\title{
The importance of surgical margins in malignant Triton tumour of the trunk and extremities
}

\author{
PANAGIOTIS TSAGOZIS ${ }^{1-3}$, CZAR LOUIE GASTON ${ }^{1,4}$, FELIX HAGLUND ${ }^{5}$, JAN BALKO ${ }^{6}$, \\ VAIYAPURI SUMATHI ${ }^{6}$, ROBERT GRIMER ${ }^{1}$ and MICHAEL PARRY ${ }^{1}$ \\ ${ }^{1}$ The Royal Orthopaedic Hospital Oncology Service, Royal Orthopaedic Hospital, Birmingham B31 2AP, UK; \\ ${ }^{2}$ Musculoskeletal Tumour Service, Karolinska University Hospital, 17176 Stockholm; \\ ${ }^{3}$ Department of Molecular Medicine and Surgery, Karolinska Institutet, 17177 Stockholm, Sweden; \\ ${ }^{4}$ University of the Philippines, Quezon City 1101, Philippines; ${ }^{5}$ Department of Oncology-Pathology, Karolinska Institutet, \\ 17177 Stockholm, Sweden; ${ }^{6}$ Department of Musculoskeletal Pathology, \\ Royal Orthopaedic Hospital NHS Foundation Trust, Birmingham B31 2AP, UK
}

Received July 23, 2020; Accepted October 21, 2020

DOI: $10.3892 / \mathrm{ol} .2020 .12381$

\begin{abstract}
Malignant Triton tumour (MTT) is a rare variant of malignant peripheral nerve sheath tumour with partial rhabdomyosarcomatous differentiation. To the best of our knowledge, the importance of the surgical resection margins on the outcome of patients with MTT is unknown. The present study is a retrospective review of 24 patients treated for MTT of the trunk and the extremities between 1997 and 2015 in two institutions. The association of surgical margins with overall and tumour recurrence-free survival was analysed. Furthermore, the typical morphological and immunohistochemical characteristics of the tumour were described. In patients treated with curative intent (17/24), a surgical margin exceeding $1 \mathrm{~mm}$ was significantly associated with better overall survival and local recurrence-free survival. The oncological outcome was however poor, with only $28 \%$ of patients surviving at 5 years. Histopathologically, necrosis was a common feature, and most tumours displayed focal positivity for S100 protein and focal or strong positivity for desmin. The present results highlight the aggressive behaviour of MTTs and underline the importance of adequate surgical treatment.
\end{abstract}

\section{Introduction}

Malignant peripheral nerve sheath tumour (MPNST) with rhabdomyoblastic differentiation, otherwise known as malignant Triton tumour (MTT), is a rare entity. It derives its name from early experiments with the amphibian Triton, which is known to regenerate limbs containing muscle upon ectopic

Correspondence to: Dr Panagiotis Tsagozis, Musculoskeletal Tumour Service, Karolinska University Hospital, Solna, 17176 Stockholm, Sweden

E-mail: panagiotis.tsagkozis@sll.se

Key words: sarcoma, Triton, surgery, margin, survival, recurrence implantation of the cut end of the sciatic nerve $(1,2)$. In a murine model, microenvironment-specific transformation of muscle-derived stem/progenitor cells has been shown to drive the formation of MTTs (3).

The presentation, natural history and response to treatment of MTTs are largely unknown, since the published data are confined to case reports and small patient series. Many MTTs arise in the context of type- 1 neurofibromatosis (von Recklinghausen's disease), and patients generally have a poor prognosis $(4,5)$.

It appears that complete surgical excision of MTT confers a survival benefit and adjuvant radiotherapy is important in the treatment of the disease (6). No study has yet proven any correlation between the quality of surgical margins and treatment outcome. This is probably due to the fact that results were derived from small case series, or review of heterogeneous case-reports.

In the present article, we describe the clinical presentation, treatment, and oncologic outcome of 24 patients diagnosed with MTT of the trunk and the extremities, with emphasis on the effect of surgical margins. The morphological and immunohistochemical profile of the tumours is also described.

\section{Materials and methods}

Study design. This is a two-institution study, of the Karolinska University Hospital, Stockholm, Sweden and the Royal Orthopaedic Hospital, Birmingham, UK, which complied to the ethical guidelines of the Royal Orthopaedic Hospital NHS Trust, and the relevant ethical permit number of the Karolinska University Hospital and Karolinska Institutet is 2013/1979-31. The study population was derived from a retrospective analysis of prospectively collected databases from the two independent institutions and consisted of 24 patients with MTT of the trunk or extremities diagnosed and treated between 1997 and 2015.

Patient characteristics, diagnostic procedures and treatment. There were 13 male and 11 female patients. The median age was 57 years (range 9-87 years). The median duration of symptoms 
was 18 months (range 6-520 months). In 6 patients, there was a history of type 1 neurofibromatosis. Five patients presented with lung metastases and 1 with lymph node metastasis at diagnosis. The tumour was localized in the lower extremity in 12 patients, the pelvis in 7, upper extremity in 3 and trunk in 2 .

All patients underwent local staging in the form of magnetic resonance imaging (MRI) and distant staging comprised plain radiography or computed tomography scan of the chest. Pre-treatment biopsy of the lesion was performed, and diagnosis and treatment decisions were made following discussion at a multidisciplinary team conference.

Twenty-one patients had surgical treatment. This entailed local excision in 18 cases and amputation in 3 ( 1 hindquarter amputation and 2 hip disarticulations). The mean volume of the surgically resected tumours was $0,6 \mathrm{dm}^{3}$ (range 0,05-10 dm $\mathrm{dm}^{3}$ ). Grading was according to the FNCLCC classification, and was recorded for 18 tumours. Of these, 1 was low-grade (grade 1), whereas 17 were high-grade neoplasms (3 grade 2 and 14 grade 3). Surgical specimens were marked with orientation sutures and transported fresh to the pathology laboratory, without any mechanical stabilization of the specimen. The evaluation of surgical margins was done by experienced musculoskeletal pathologists, without the presence of the surgeon, and margins were recorded in millimetres, also reporting the nature of the tissue forming the margins. They were subsequently analysed according to the Enneking classification, the American Joint Committee on Cancer (AJCC) residual tumour classification ( $\mathrm{R}$ classification) and the $\mathrm{R}+1$ classification proposed by the Union International Côntre le Cancer (UICC) (7-9).

Morphological evaluation was done using haematoxylin and eosin staining. Paraffin blocks were processed in a Varistain Gemini stainer. Sections were passed through xylene (Genta Medical) for $2 \mathrm{~min}+1 \mathrm{~min}+1 \mathrm{~min}$ and then underwent sequential alcohol solutions $100-95-80 \%$ (3 min each) and then washed with running tap water for 2 min. Sections were then stained in Ehrlich's haematoxylin (Cell Path) for $12 \mathrm{~min}$, washed in running tap water for $2 \mathrm{~min}$, processed in acid alcohol for $8 \mathrm{sec}$ and washed again in running tap water for $5 \mathrm{~min}$. They were then stained in 1\% aqueous eosin (Atom Scientific) for $2 \mathrm{~min}$, washed in running tap water for $30 \mathrm{sec}$ and processed through serial alcohol $(3 \times 30 \mathrm{sec})$ and xylene solutions (30 sec $+2 \times 1 \mathrm{~min})$.

The Dako Ventana Ultra platform was used for immunohistochemistry. For desmin staining, samples were pre-treated in cell conditioning 1 (CC1, Ventana Medical Systems) for $8 \mathrm{~min}$, incubated at $37^{\circ} \mathrm{C}$ for $4 \mathrm{~min}$, and then enzyme treated with protease for $18 \mathrm{~min}$, following treatment with anti-desmin antibody (Dako) for $16 \mathrm{~min}$. Sections were then stained with haematoxylin for $8 \mathrm{~min}$ and bluing reagent for $4 \mathrm{~min}$. For EMA staining, $\mathrm{CC1}$ pretreatment was carried out for $64 \mathrm{~min}$ and the incubated with anti-EMA antibody (Dako) for $32 \mathrm{~min}$ and incubated at $37^{\circ} \mathrm{C}$ for 4 min before being stained with haematoxylin for $8 \mathrm{~min}$ and bluing reagent for $4 \mathrm{~min}$. For CD34 staining, there was no pre-treatment with $\mathrm{CC} 1$, sections were incubated at $37^{\circ} \mathrm{C}$ for 4 min and then with anti-CD34 antibody (Dako) for $8 \mathrm{~min}$, following staining with haematoxylin for $8 \mathrm{~min}$ and bluing reagent for $4 \mathrm{~min}$. For HMB45 staining, CC1 pretreatment was carried out for $36 \mathrm{~min}$, followed by incubation at $37^{\circ} \mathrm{C}$ for $4 \mathrm{~min}$ and incubation with anti-HMB45 for
$8 \mathrm{~min}$, before final staining with haematoxylin for $8 \mathrm{~min}$ and bluing reagent for $4 \mathrm{~min}$. All other steps were performed at room temperature and analysis was done by light microscopy.

Three patients did not have surgical treatment: 2 had lung metastases at presentation and were treated with local radiotherapy and/or systemic chemotherapy, and 1 patient presented with a massive tumour arising from the thigh and extending intra-abdominally to the liver and had only palliative treatment.

Data regarding the use of radiotherapy were available for 16 of the 18 patients treated with limb-sparing surgery: 14 patients received radiotherapy with a median dose of $60 \mathrm{~Gy}$ (20-66 Gy), whereas 2 patients did not receive radiotherapy due to significant comorbidities. Eight out of 24 patients had chemotherapy, 2 in a neoadjuvant setting and 6 for metastatic disease or unresectable local recurrence. Doxorubicin/ifosfamide was used in 3 patients, adriamycin in 2, cisplatin/doxorubicin in 1 , and vincristine/ifosfamide/actinomycin in 1. Data regarding the given chemotherapy regimen were missing for 1 patient.

Follow-up was according to routine sarcoma surveillance guidelines and median duration of follow up was 24 months (range 1-174 months).

Statistical analysis. Statistical analysis was carried out using the SPSS software (version 20, SPSS Inc.). The Kaplan-Meier technique was used for survival analysis with difference in survival between groups compared using the log-rank test. Comparisons between categorical variables were done using Pearson's Chi-square test. All tests were double-sided, and a $\mathrm{P} \leq 0.05$ was considered significant.

\section{Results}

Oncologic outcome. Of the 24 patients, 17 had no metastases at presentation and their tumours were operable, and treatment therefore was considered with a curative intent. Median overall survival (OS) of patients treated in a curative setting was 35 months (95\% CI 16-54 months), and OS at 5 years was $32 \%$. Seven of these 17 patients developed metastatic lung disease at a median of 9 months after diagnosis (range 7-22 months) and 2 developed a local recurrence, at 14 and 123 months after index surgery. Median local recurrence-free survival (LRFS) for patients treated with curative intent was 35 months $(95 \% \mathrm{CI}$ 18-52 months), and LRFS at 5 years was $32 \%$.

Of the remaining 7 patients, 6 presented with metastatic disease at diagnosis (5 with lung metastases and 1 with lymph node metastasis), and 1 had a very large, inoperable tumour, and were thus considered as treated with non-curative (palliative) intent. Four of these 7 patients had surgical resection of the primary tumour. The median OS of patients treated in a palliative setting was 13 months, and OS at 5 years was $18 \%$.

Median OS of all 24 patients was 25 months, and OS at 5 years was $28 \%$. Median LRFS of the entire cohort was 24 months and LRFS at 5 years was $23 \%$. The difference between OS between patients treated in a curative and a palliative setting did not reach statistical significance $(\mathrm{P}=0.127)$, but there was a significant difference in LRFS $(\mathrm{P}<0.001)$ (Fig. 1).

Effect of the quality of surgical margins on outcome. In patients treated with a curative intent, surgical margins classified as per Enneking were known for 15 patients and were wide in 1 patient, 

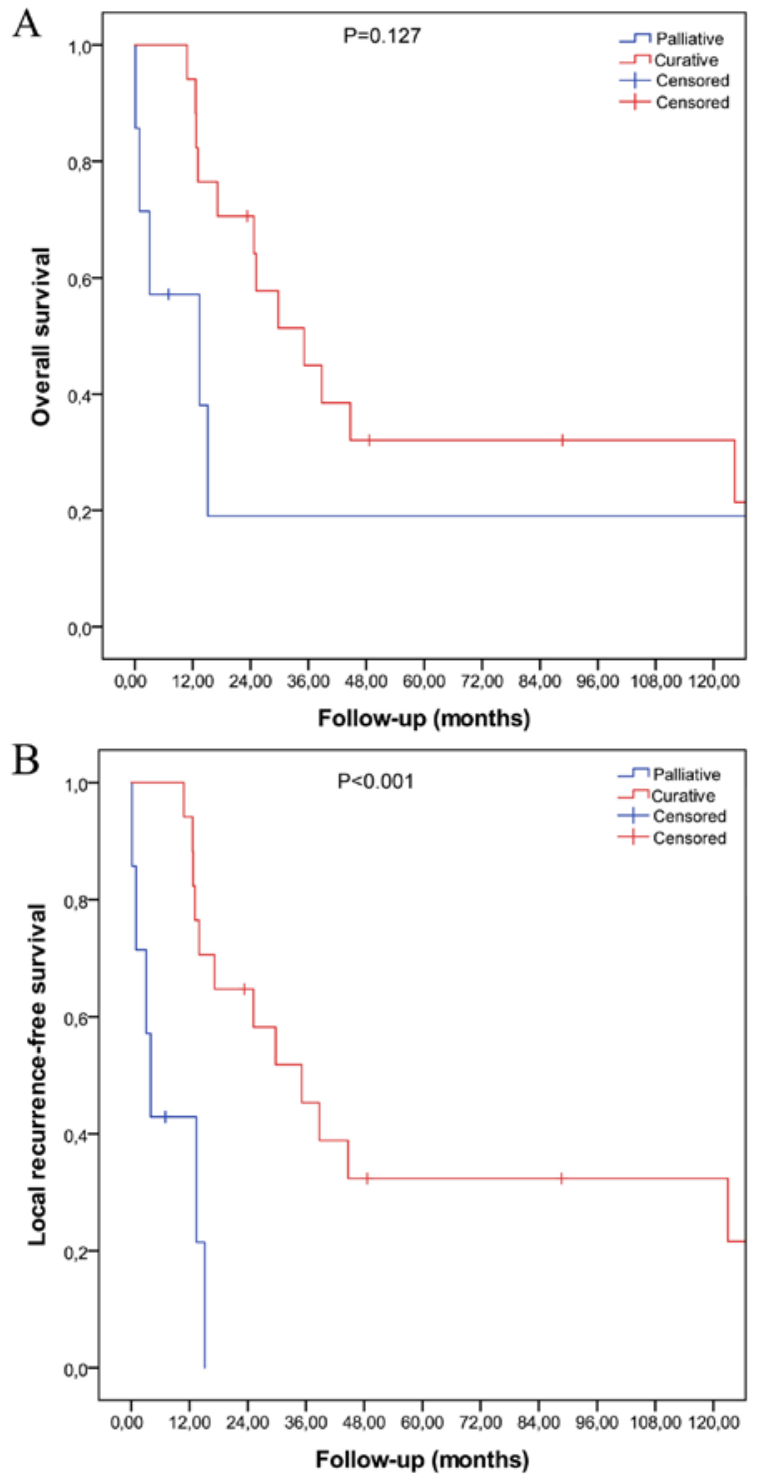

Figure 1. Kaplan-Meier analysis for (A) overall survival and local (B) recurrence-free survival of 24 patients with malignant Triton tumours of the trunk and the extremities. Patients treated in a curative intention had superior local recurrence-free survival, $(\mathrm{P}<0.001)$ while the difference in overall survival did not reach statistical significance $(\mathrm{P}=0.127)$.

marginal in 12 and intralesional in 2. Margins were not known for 2 patients, a 71-year old man with a tumour of the posterior thigh and an 11-year-old girl operated for a MTT of the cubital fossa. The former developed local recurrence 10 years after the operation and succumbed to the disease shortly after and the latter developed metastatic disease 2 years after surgery and died 4 years after primary surgery. Of the 12 patients with marginal margins, 6 were considered as clear (over $1 \mathrm{~mm}$ ) and 6 clear but close $(0-1 \mathrm{~mm})$. The quality of surgical margins correlated to the oncologic outcome of patients treated with curative intent. Analysis using the Enneking classification was not meaningful as only 1 patient had wide margins. Therefore, we concentrated our analysis on the $\mathrm{R}$ and $\mathrm{R}+1$ classifications. We observed that patients with clear but close margins (less than $1 \mathrm{~mm}$ ) had similar outcome to the ones with intralesional ones regarding LRFS and OS (Fig. 2). A resection margin greater than $1 \mathrm{~mm}(\mathrm{R}+1 \mathrm{~mm})$ was however, associated with
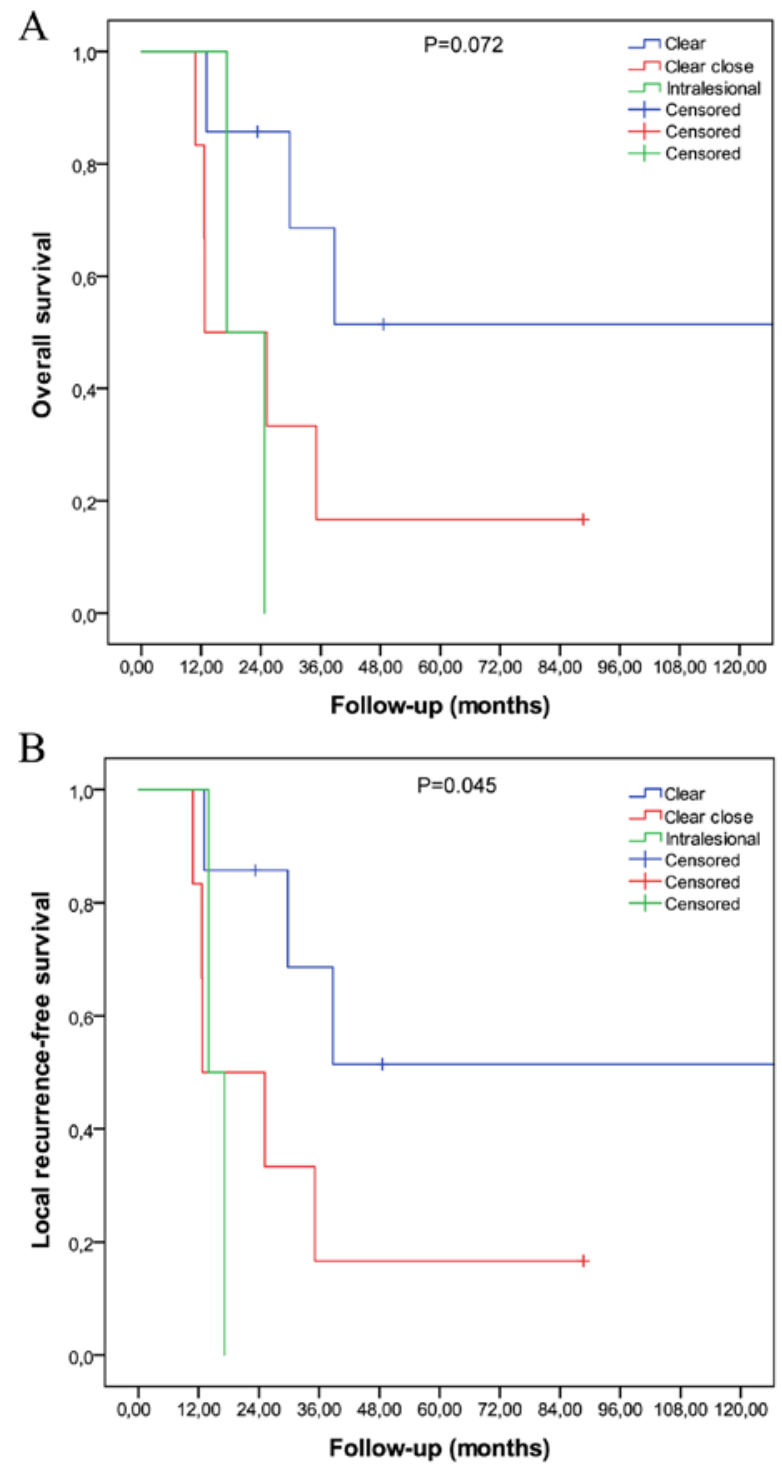

Figure 2. Effect of surgical margins on survival as per Kaplan-Meier for patients with malignant Triton tumours of the trunk and the extremities treated in a curative intention. A clear surgical margin below $1 \mathrm{~mm}(\mathrm{R} 0$, clear close) is comparable to an intralesional one, and inferior to a clear (R0+1 mm) margin regarding (A) overall and (B) local recurrence-free survival.

an improved OS and LRFS (Fig. 3). The effect of the quality of surgery was stronger than established prognostic factors for soft-tissue sarcomas such as volume and grade (Table I).

Morphological and histopathological profile of the tumours. MTT commonly displayed extensive geographic necrosis. The rhabdomyoblastic differentiation was usually a minority component and was characterized by polygonal epithelioid cells with copious, brightly eosinophilic cytoplasm (Fig. 4A). The morphological and immunohistochemical profile of the tumours could be retrieved for 11 cases (Table II). Necrosis was present in most specimens, whereas the mitotic activity was variable. Most tumours displayed focal positivity for S100 protein. Focal or strong positivity for desmin was noted for 8/11 tumours (Fig. 4B). A focal staining pattern for EMA was observed in 3/11 and a strong staining pattern was seen in $1 / 11$ of the specimens. Three out of 11 tumours stained positive for CK. All tumours were negative for CD34 and HMB45. 
Table I. Survival analysis of possible prognostic factors on OS as well as LRFS of patients diagnosed with malignant Triton tumours of the trunk and the extremities as per Kaplan-Meier. Mean survival time in months for each group is shown, with 95\% CI of the mean survival time in brackets, and P-values for comparison between groups were determined according to the log-rank test.

\begin{tabular}{|c|c|c|c|c|}
\hline Features & $\begin{array}{c}\text { OS, months } \\
\text { (mean, } 95 \% \mathrm{CI})\end{array}$ & P-value & $\begin{array}{l}\text { LRFS, months } \\
(\text { mean, } 95 \% \mathrm{CI})\end{array}$ & P-value \\
\hline Age, years & & 0.179 & & 0.453 \\
\hline$\leq 57$ & $114(42-186)$ & & $92(23-161)$ & \\
\hline$>57$ & $38(12-64)$ & & $38(12-64)$ & \\
\hline Volume, $\mathrm{dm}^{3}$ & & 0.126 & & 0.340 \\
\hline$\leq 0.6$ & $25(17-35)$ & & $25(15-34)$ & \\
\hline$>0.6$ & $92(37-147)$ & & $69(15-123)$ & \\
\hline Grade (FNCLCC) & & 0.450 & & 0.339 \\
\hline 2 & $70(0-154)$ & & $66(0-153)$ & \\
\hline 3 & $61(10-113)$ & & $40(0-81)$ & \\
\hline Clear surgical margin $(\mathrm{R}+1 \mathrm{~mm})$ & & 0.049 & & 0.045 \\
\hline Yes & $152(56-48)$ & & $151(56-248)$ & \\
\hline No & $28(12-45)$ & & $27(10-44)$ & \\
\hline
\end{tabular}

The effects of surgical margins and of radiotherapy were evaluated in patients treated in a curative intent. OS, overall survival; LRFS, local recurrence-free survival.

Table II. Histopathological analysis of samples from 11 of the 24 patients with malignant Triton tumours of the trunk and the extremities.

\begin{tabular}{|c|c|c|c|c|c|c|c|c|c|c|c|c|}
\hline Patient & $\begin{array}{l}\text { Age, } \\
\text { years }\end{array}$ & Sex & Grade & $\begin{array}{c}\text { Necrosis, } \\
\%\end{array}$ & $\begin{array}{l}\text { Mitoses, } \\
\text { /10 hpf }\end{array}$ & S100 & Desmin & CD34 & EMA & SMA & CK & HMB45 \\
\hline 1 & 77 & $\mathrm{~F}$ & 1 & 0 & 3 & 0.5 & 0.5 & 0 & 0 & 0 & 0 & 0 \\
\hline 2 & 57 & M & 3 & 20 & 15 & 0.5 & 0.5 & 0 & 0 & 0 & 0 & 0 \\
\hline 3 & 33 & M & 3 & 20 & 37 & 0 & 1 & 0 & 0 & 0 & 0 & 0 \\
\hline 4 & 51 & $\mathrm{~F}$ & 2 & 0 & 5 & 0.5 & 0.5 & 0 & 0 & 0 & 0 & 0 \\
\hline 5 & 69 & M & 3 & 50 & 30 & 0.5 & 1 & 0 & 0 & 0 & 0 & N/A \\
\hline 6 & 10 & M & 2 & N/A & 4 & 0.5 & 1 & 0 & 0.5 & 0 & 0.5 & N/A \\
\hline 7 & 39 & M & 3 & 20 & 20 & 0.5 & N/A & 0 & 0 & 0 & 0 & 0 \\
\hline 8 & 73 & $\mathrm{~F}$ & 3 & 50 & 100 & 0.5 & 1 & 0 & 1 & 0 & 0.5 & 0 \\
\hline 9 & 79 & $\mathrm{~F}$ & 3 & 50 & 15 & 0.5 & 0 & N/A & 0.5 & 0 & 0 & 0 \\
\hline 10 & 85 & M & 2 & N/A & N/A & 0.5 & 0.5 & N/A & 0 & 0.5 & 0.5 & N/A \\
\hline 11 & 54 & M & 3 & N/A & 15 & 0.5 & 0 & 0 & 0.5 & 0 & 0 & 0 \\
\hline
\end{tabular}

0, no staining, 0.5 , focal staining, 1 , strong staining. N/A, not available; F, female, M, male; hpf, high-power field.

\section{Discussion}

MTT are rare soft tissue sarcomas characterised by morphological appearances of MPNST with associated rhabdomyoblastic differentiation. Evidence within the literature is based predominantly on case reports and small case series, but appears to show that complete surgical resection and use of radiotherapy are associated with a superior prognosis (6). However, given the paucity of available evidence, it is felt that these observations are largely explained by selection bias. Our study has significant limitations: it is retrospective in nature, and thus also suffers from selection bias in treatment decisions. Furthermore, the number of patients included is limited and the study extends over a long time-period Both shortcomings of the study are explained by the rarity of this condition.

We have demonstrated that the quality of surgical margins is important. In patients who underwent resection with curative intent, those that had surgical margins of at least $1 \mathrm{~mm}$, corresponding to $\mathrm{R} 0$ resection in the $\mathrm{R}+1$ classification, had a better OS and LRFS when compared to those with margins less than $1 \mathrm{~mm}$ or an intralesional margin. The observation that close surgical margins (less than $1 \mathrm{~mm}$ ) were inferior 

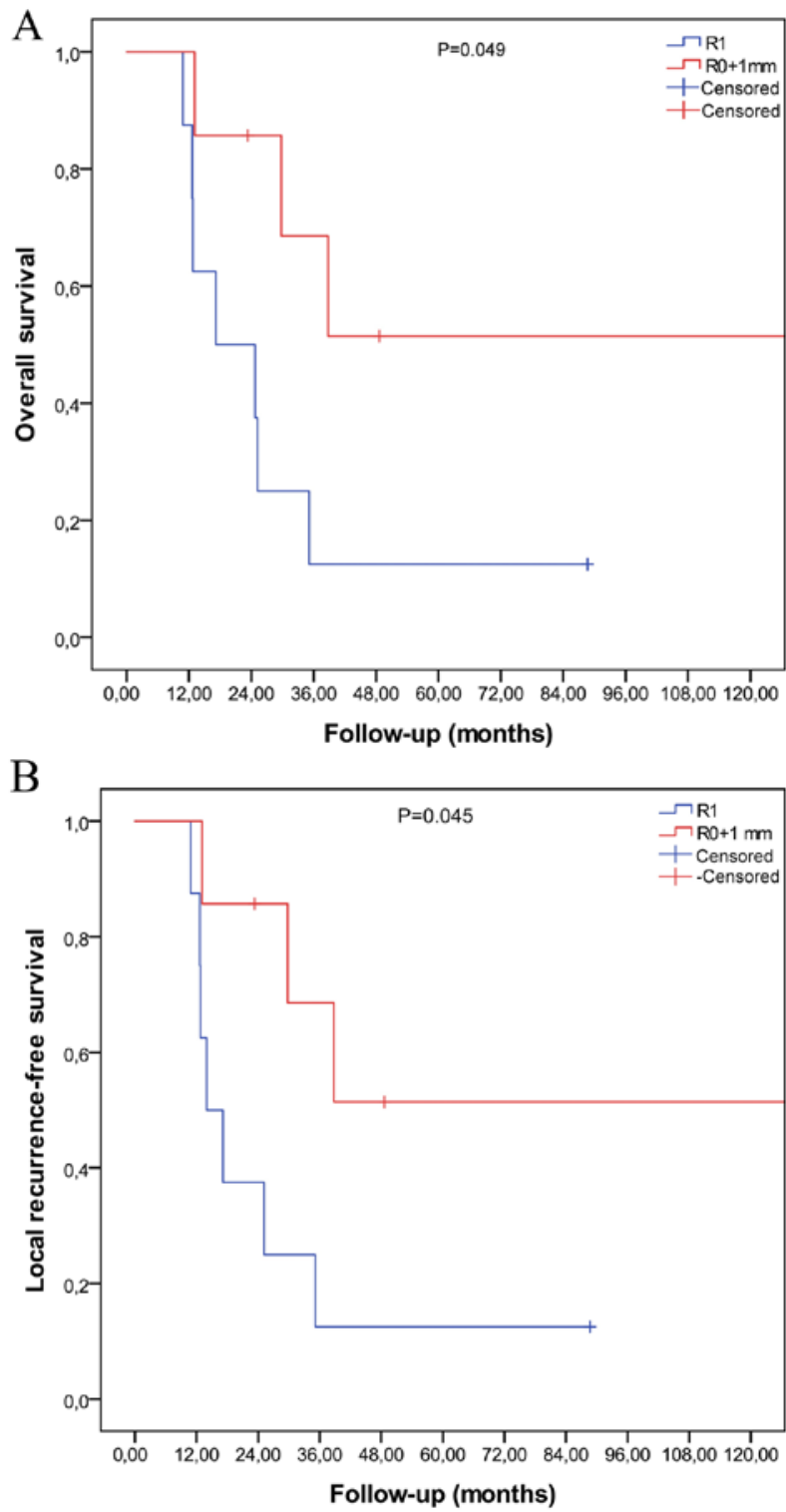

Figure 3. Resection with clear margins of at least $1 \mathrm{~mm}$ was associated with superior (A) overall survival $(\mathrm{P}=0.049)$ and $(\mathrm{B})$ local recurrence-free survival $(\mathrm{P}=0.045)$ among patients with malignant Triton tumours treated in a curative intention.

may be due to fact that in cases of large tumours with an infiltrative growth pattern, such as those in our series, a small intralesional area may be missed and misinterpreted as a close clear margin. We focused on the role of the quality of surgery since radiotherapy was routinely given after limb-sparing surgery in this series.

The high number of patients presenting with metastases at diagnosis and the high risk for secondary metastatic disease in our cohort are notable and underline the aggressive behaviour of this tumour. Small heterogenous studies have shown that patients with MTTs have a poor prognosis, clearly inferior to patients with conventional MPNST $(6,10)$, where overall survival is seen to be in the region of $50 \%$ at 5 years (11), almost twice as good as the $28 \% 5$-year survival seen in this series.

The immunohistochemical profile of MTT has also not been described in detail thus far. As expected, the often focal
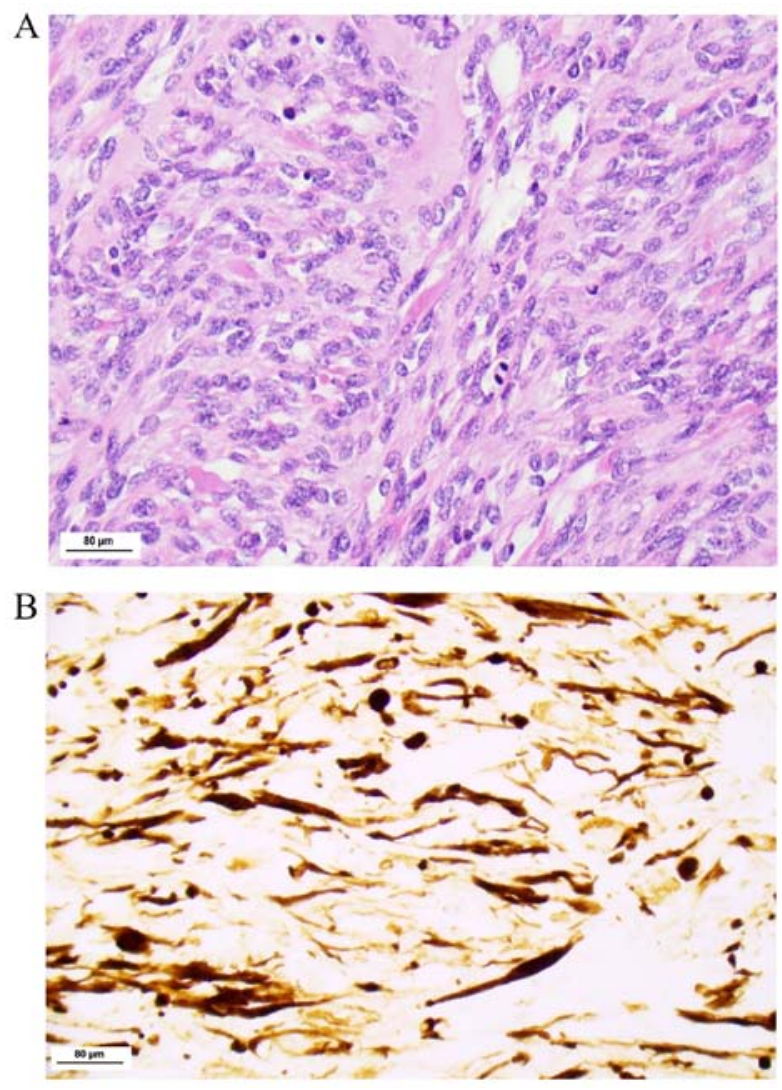

Figure 4. Morphological and immunohistochemical pattern of malignant Triton tumours. (A) Haematoxylin and eosin staining showing typical rhabdomyoblasts and (B) immunohistochemistry showing positivity for desmin. A $80 \mu \mathrm{m}$ scale is shown in both images.

rhabdoid differentiation could be confirmed with immunoreactivity for desmin establishing a diagnosis of MTT. Similar to MPNST, immunoreactivity for the S-100 protein was weak and focal in most cases, corroborating previous findings (12). A minority of the tumours expressed the epithelial markers CK and EMA, implying that some tumours can have areas of epithelial differentiation, a phenomenon that has also been described in MPNSTs $(13,14)$. None of the tumours in our series stained positive for HMB45, a typical marker for melanomas and PEComas, or CD34, a protein mainly expressed by cells of the haematopoietic line and endothelium.

Overall, our findings strengthen the view that surgery remains the mainstay of treatment for MTTs $(6,15)$. Patients with malignant Triton tumour of the trunk and the extremities have a poor prognosis, with a high rate of metastatic disease. Local resection with a surgical margin of at least $1 \mathrm{~mm}$ is important for the outcome of curative treatment.

\section{Acknowledgements}

The authors would like to thank Mr. Hamed Ali, (Department of Musculoskeletal Pathology, University Hospitals Birmingham) for their technical support.

\section{Funding}

No funding was received. 


\section{Availability of data and materials}

The data used in this study are not publicly available to protect patient integrity, but anonymised data are available from the corresponding author upon request.

\section{Authors' contributions}

PT performed data collection from the databases of both institutions, analysis and manuscript writing. CLG performed data collection from the Royal Orthopaedic Hospital database and edited the manuscript. FH conducted data collection from the Karolinska University Hospital database, analysis and manuscript writing. JB and VS contributed to the conception of the study, collected, analysed and interpreted data from the Laboratory of Musculoskeletal Pathology of the Royal Orthopaedic Hospital, and wrote and revised the manuscript. RG and MP conceived and designed the study, analysed and interpreted data, drafted and revised the manuscript. All authors have read and approved the final manuscript.

\section{Ethics approval and consent to participate}

This is a review study from prospectively collected databases that confirmed to Institutional Review Board requirements. The study complied with the ethical guidelines of the Royal Orthopaedic Hospital NHS Trust and the relevant ethical permit number of the Karolinska University Hospital and Karolinska Institutet is 2013/1979-31. Data are presented anonymised and due to the nature of the study patient consent is not applicable.

\section{Patient consent for publication}

Not applicable.

\section{Competing interests}

The authors declare that they have no competing interests.

\section{References}

1. Woodruff JM, Chernik NL, Smith MC, Millett WB and Foote FW Jr: Peripheral nerve tumors with rhabdomyosarcomatous differentiation (malignant 'Triton' tumors). Cancer 32: 426-439, 1973
2. Woodruff JM and Perino G: Non-germ-cell or teratomatous malignant tumors showing additional rhabdomyoblastic differentiation, with emphasis on the malignant Triton tumor. Semin Diagn Pathol 11: 69-81, 1994

3. Lavasani M, Pollett JB, Usas A, Thompson SD, Pollett AF and Huard J: The microenvironment-specific transformation of adult stem cells models malignant triton tumors. PLoS One 8: e82173, 2013.

4. Aldlyami E, Dramis A, Grimer RJ, Abudu A, Carter SR and Tillman RM: Malignant triton tumour of the thigh - a retrospective analysis of nine cases. Eur J Surg Oncol 32: 808-810, 2006.

5. Stasik CJ and Tawfik O: Malignant peripheral nerve sheath tumor with rhabdomyosarcomatous differentiation (malignant triton tumor). Arch Pathol Lab Med 130: 1878-1881, 2006.

6. McConnell YJ and Giacomantonio CA: Malignant triton tumors--complete surgical resection and adjuvant radiotherapy associated with improved survival. J Surg Oncol 106: 51-56, 2012.

7. Enneking WF, Spanier SS and Goodman MA: A system for the surgical staging of musculoskeletal sarcoma. Clin Orthop Relat Res: 153, 106-120, 1980.

8. AJCC Manual for Staging of Cancer. 3rd edition. JB Lippincott, Philadelphia, 1988.

9. Wittekind C, Compton CC, Greene FL and Sobin LH: TNM residual tumor classification revisited. Cancer 94: 2511-2516, 2002.

10. Kamran SC, Howard SA, Shinagare AB, Krajewski KM, Jagannathan JP,Hornick JL and Ramaiya NH: Malignant peripheral nerve sheath tumors: Prognostic impact of rhabdomyoblastic differentiation (malignant triton tumors), neurofibromatosis 1 status and location. Eur J Surg Oncol 39: 46-52, 2013.

11. Porter DE, Prasad V, Foster L, Dall GF, Birch R and Grimer RJ: Survival in malignant peripheral nerve sheath tumours: A comparison between sporadic and neurofibromatosis type 1-associated tumours. Sarcoma 2009: 756395, 2009.

12. Daimaru Y, Hashimoto H and Enjoji M: Malignant 'triton' tumors: A clinicopathologic and immunohistochemical study of nine cases. Hum Pathol 15: 768-778, 1984.

13. Klijanienko J, Caillaud J-M,Lagacé R and Vielh P: Cytohistologic correlations of 24 malignant peripheral nerve sheath tumor (MPNST) in 17 patients: The Institut Curie experience. Diagn Cytopathol 27: 103-108, 2002.

14. Kurtkaya-Yapicier O, Scheithauer BW, Woodruff JM, Wenger DD, Cooley AM and Dominique D: Schwannoma with rhabdomyoblastic differentiation: A unique variant of malignant triton tumor. Am J Surg Pathol 27: 848-853, 2003.

15. Li G, Liu C, Liu Y, Xu F, Su Z, Wang Y, Ren S, Deng T, Huang D, Tian Y, et al: Analysis of clinical features and prognosis of malignant triton tumor: A report of two cases and literature review. Oncol Lett 10: 3551-3556, 2015. 